\title{
CRITERIA FOR DISCRETE SPECTRUM OF SINGULAR SELFADJOINT DIFFERENTIAL OPERATORS
}

\author{
L. W. ROLLINS
}

\begin{abstract}
Under certain conditions on the coefficients of symmetric singular differential operators of order $2 n$, selfadjoint extensions are shown to have a discrete spectrum. The results are proven specifically for the Friedrichs extension.
\end{abstract}

1. Introduction. Consider the formally selfadjoint differential operator defined by

$$
\tau u(x)=\frac{1}{m(x)} \sum_{k=0}^{n}(-1)^{k} D^{k} p_{k}(x) D^{k} u(x), \quad a \leqq x<b,
$$

where $b$ may be finite or infinite, $p_{k}$ is real-valued, nonnegative, and in $C^{(k)}[a, b)$ for $0 \leqq k \leqq n$, and, for $x \in[a, b), p_{n}(x)>0$, and $m(x)>0$. The endpoint $b$ is possibly a singular endpoint.

Let $L^{2}(m)$ be the Hilbert space of all measurable functions, $f$, defined on $(a, b)$ for which $\int_{a}^{b}|f(x)|^{2} m(x) d x<\infty$, with inner product and norm denoted by $(\cdot, \cdot)$ and $\|\cdot\|$ respectively. Let $T_{0}$ be the symmetric linear operator defined by $T_{0} u=\tau u$ whose domain $D\left(T_{0}\right)$ consists of those functions in $L^{2}(m)$ which have $2 n$ continuous derivatives and compact support in $(a, b)$. Then $D\left(T_{0}\right)$ is dense in $L^{2}(m)$. Under these conditions $T_{0}$ will have a selfadjoint extension; in particular, it will have the Friedrichs extension [6, p. 124].

This paper is concerned with finding conditions on the functions $p_{k}$, $1 \leqq k \leqq n$, which guarantee that the Friedrichs extension has a compact inverse and, hence, a discrete spectrum. Since all selfadjoint extensions of $T_{0}$ have the same continuous spectrum [5, Theorem 2, p. 92], we conclude that every selfadjoint extension of $T_{0}$ has a discrete spectrum.

Early investigations were confined to second order differential operators with the weight function $m(x)=1$ and are available in the literature (see for example: Naĭmark [5, Chapter VII], and Dunford-Schwartz [2, Chapter XIII]). Recently Eastham [3] obtained results for higher order

Presented to the Society, November 19, 1971; received by the editors August 10, 1971 and, in revised form, November 19, 1971.

AMS 1970 subject classifications. Primary 34B25, 47E05; Secondary 47A20, 47B25.

Key words and phrases. Singular differential operators, selfadjoint extensions, spectrum.

(c) American Mathematical Society 1972 
differential operators with $b=\infty$. The results here will be compared with Eastham's and, also, earlier results of Friedrichs [4]. The techniques are an extension of those used by Baxley [1].

2. Statement of theorem. The operator $T_{0}$ is symmetric and semibounded from below. To see the latter, use repeated integration by parts and the fact that $p_{k} \geqq 0$ for $0 \leqq k \leqq n$, to get

$$
\left(T_{0} u, u\right)=\int_{a}^{b} \sum_{k=0}^{n} p_{k}(x)\left[u^{(k)}(x)\right]^{2} d x \geqq 0
$$

for $u \in D\left(T_{0}\right)$. Therefore $T_{0}$ has a Friedrichs extension, $T$.

Let $k$ be an integer, $1 \leqq k \leqq n$. For this $k$ define a sequence of functions $\left\{h_{i}\right\}_{1}^{k}$ as follows:

If, for each $y$ in $[a, b)$, it is true that $\int_{y}^{b} 1 / p_{k}(t) d t$ is finite, then

Otherwise

$$
h_{1}(x, k)=\left(\int_{x}^{b} \frac{1}{p_{k}(t)} d t\right)^{1 / 2}, \text { for } x \in[a, b) .
$$

$$
h_{1}(x, k)=\left(\int_{a}^{x} \frac{1}{p_{k}(t)} d t\right)^{1 / 2}, \text { for } x \in[a, b) .
$$

If $i$ is any integer $1 \leqq i \leqq k-1$, and if, for each $y$ in $[a, b)$, it is true that $\int_{y}^{b} h_{i}(t, k) d t$ is finite, then

Otherwise

$$
h_{i+1}(x, k)=\int_{x}^{b} h_{i}(t, k) d t, \quad \text { for } x \in[a, b) .
$$

$$
h_{i+1}(x, k)=\int_{a}^{x} h_{i}(t, k) d t, \text { for } x \in[a, b) .
$$

THEOREM. Let $T$ be the Friedrichs extension of $T_{0}$. If

$$
\int_{a}^{b} m(x)\left[h_{k}(x, k)\right]^{2} d x=M<\infty
$$

for at least one integer $k, 1 \leqq k \leqq n$, then $T$ has a compact inverse, and in this case, every selfadjoint extension of $T_{0}$ has a discrete spectrum.

3. Proof of theorem. We first prove two lemmas.

LEMMA 1. If $u \in D\left(T_{0}\right)$ and (3) is satisfied for some $k, 1<k \leqq n$, then for $a \leqq x_{1}<x_{2}<b$,

$$
\left|u\left(x_{2}\right)-u\left(x_{1}\right)\right| \leqq\left(T_{0} u, u\right)^{1 / 2} \int_{x_{1}}^{x_{2}} h_{k-1}(t, k) d t .
$$


Proof. For $u \in D\left(T_{0}\right)$, it follows from the Schwartz inequality

$$
\begin{aligned}
\left|u^{(k-1)}\left(x_{2}\right)-u^{(k-1)}\left(x_{1}\right)\right|^{2} & \leqq\left|\int_{x_{1}}^{x_{2}} u^{(k)}(t) d t\right|^{2} \\
& \leqq \int_{x_{1}}^{x_{2}} p_{k}(t)\left[u^{(k)}(t)\right]^{2} d t \int_{x_{1}}^{x_{2}} \frac{1}{p_{k}(t)} d t \\
& \leqq\left(T_{0} u, u\right) \int_{x_{1}}^{x_{2}} \frac{1}{p_{k}(t)} d t
\end{aligned}
$$

Now setting $x_{1}=x$ and $x_{2}=b$, or $x_{1}=a$ and $x_{2}=x<b$ for $h_{1}(x, k)$ defined as in (1a) or (1b) respectively, leads to the following:

$$
\begin{aligned}
\left|u^{(k-1)}(x)\right|^{2} & \leqq\left(T_{0} u, u\right)\left[h_{1}(x, k)\right]^{2}, \\
\left|u^{(k-1)}(x)\right| & \leqq\left(T_{0} u, u\right)^{1 / 2} h_{1}(x, k) .
\end{aligned}
$$

Using (4) above, we obtain

$$
\begin{aligned}
\mid u^{(k-2)}\left(x_{2}\right) & -u^{(k-2)}\left(x_{1}\right) \mid \\
& =\left|\int_{x_{1}}^{x_{2}} u^{(k-1)}(t) d t\right| \\
& \leqq\left(\int_{x_{1}}^{x_{2}}\left(T_{0} u, u\right)^{1 / 2} h_{1}(t, k) d t\right)=\left(T_{0} u, u\right)^{1 / 2}\left(\int_{x_{1}}^{x_{2}} h_{1}(t, k) d t\right) .
\end{aligned}
$$

Setting $x_{1}=x$ and $x_{2}=b$, or $x_{1}=a$ and $x_{2}=x<b$ for $h_{2}(x, k)$ defined as in (2a) or (2b) respectively,

$$
\begin{aligned}
\left|u^{(k-2)}(x)\right|^{2} & \leqq\left(T_{0} u, u\right)\left[h_{2}(x, k)\right]^{2}, \\
\left|u^{(k-2)}(x)\right| & \leqq\left(T_{0} u, u\right)^{1 / 2} h_{2}(x, k) .
\end{aligned}
$$

Continuing by induction, we get the inequality

$$
\left|u^{(k-j)}(x)\right| \leqq\left(T_{0} u, u\right)^{1 / 2} h_{j}(x, k), \quad j=1, \cdots, k-1,
$$

in particular, for $j=k-1$,

$$
\left|u^{\prime}(x)\right| \leqq\left(T_{0} u, u\right)^{1 / 2} h_{k-1}(x, k) .
$$

Integration of both sides leads to the conclusion of the lemma.

LemMa 2. Let $T$ be the Friedrichs extension of $T_{0}$. If $u \in D(T)$ and (3) holds for some $k, 1<k \leqq n$, then for $a \leqq x_{1}<x_{2}<b$,

$$
\left|u\left(x_{2}\right)-u\left(x_{1}\right)\right| \leqq(T u, u)^{1 / 2} \int_{x_{1}}^{x_{2}} h_{k-1}(t, k) d t
$$

$$
(u, u) \leqq(T u, u) M
$$


Proof. For $u \in D(T)$ there exists a sequence $\left\{u_{m}\right\}$ with $u_{m} \in D\left(T_{0}\right)$, $m=1, \cdots$, such that $\left\|u_{m}-u\right\| \rightarrow 0$ and $\left(T_{0} u_{m}, u_{m}\right) \rightarrow(T u, u)$ as $m \rightarrow \infty$.

Since $\left(T_{0} u_{m}, u_{m}\right)$ is a convergent sequence it is bounded, that is $\left(T_{0} u_{m}, u_{m}\right) \leqq C$ for some positive number $C, m=1, \cdots$. From Lemma 1 ,

$$
\left|u_{m}\left(x_{2}\right)-u_{m}\left(x_{1}\right)\right| \leqq\left(T_{0} u_{m}, u_{m}\right)^{1 / 2} \int_{x_{1}}^{x_{2}} h_{k-1}(t, k) d t .
$$

Therefore the functions $\left\{u_{m}\right\}$ are equicontinuous and uniformly bounded on compact subsets of $[a, b)$. By the Ascoli Theorem there is a subsequence of $\left\{u_{m}\right\}$ which converges uniformly on compact subsets of $[a, b)$. Restricting attention to this subsequence and taking limits in (5) we get (a),

$$
\left|u\left(x_{2}\right)-u\left(x_{1}\right)\right| \leqq(T u, u)^{1 / 2} \int_{x_{1}}^{x_{2}} h_{k-1}(t, k) d t .
$$

For the case $h_{k}(x, k)=\int_{a}^{x} h_{k-1}(t, k) d t$, let $x_{1}=a, x_{2}=x$ in the above inequality to obtain

$$
|u(x)| \leqq(T u, u)^{1 / 2} h_{k}(x, k) .
$$

For the case $h_{k}(x, k)=\int_{x}^{b} h_{k-1}(t, k) d t$, then note that for each $u_{m}$ in the subsequence there is a $x_{2}<b$ such that $u_{m}\left(x_{2}\right)=0$. Setting $x_{1}=x$ we obtain

$$
\begin{aligned}
\left|u_{m}(x)\right| & =\left|u_{m}\left(x_{2}\right)-u_{m}(x)\right| \\
& \leqq\left(T_{0} u_{m}, u_{m}\right)^{1 / 2} \int_{x}^{x_{2}} h_{k-1}(t, k) d t \leqq\left(T_{0} u_{m}, u_{m}\right)^{1 / 2} \int_{x}^{b} h_{k-1}(t, k) d t \\
& =\left(T_{0} u_{m}, u_{m}\right)^{1 / 2} h_{k}(x, k) .
\end{aligned}
$$

Now take limits to obtain, for this case also, $|u(x)| \leqq(T u, u)^{1 / 2} h_{k}(x, k)$. Hence, in either case

$$
|u(x)|^{2} \leqq(T u, u)\left[h_{k}(x, k)\right]^{2}
$$

Integration of the above leads to (b).

Proof OF THE THEOREM. Let $\lambda$ be an eigenvalue of $T$ associated with eigenvector $u$. Then $(T u, u)=(\lambda u, u) \geqq 1 / M(u, u)$. Hence, $\lambda \geqq 1 / M>0$. Therefore all eigenvalues of $T$ are positive and $T^{-1}$ exists.

Let $\left\{T u_{n}\right\}$ be a bounded sequence in the domain of $T^{-1}$ and $K$ be a number such that $\left\|T u_{n}\right\| \leqq K<\infty, n=1, \cdots$. Then $u_{n} \in D(T)$ and, from Lemma 2(b),

$$
\left\|u_{n}\right\|^{2}=\left(u_{n}, u_{n}\right) \leqq M\left(T u_{n}, u_{n}\right) \leqq M\left\|T u_{n}\right\|\left\|u_{n}\right\|,
$$

from which follows $\left\|u_{n}\right\| \leqq M K$, and $\left(T u_{n}, u_{n}\right) \leqq M K^{2}$. 
From Lemma 2(a),

$$
\left|u_{n}\left(x_{2}\right)-u_{n}\left(x_{1}\right)\right| \leqq\left(M K^{2}\right)^{1 / 2} \int_{x_{1}}^{x_{2}} h_{k-1}(t, k) d t
$$

and, hence, $\left\{u_{n}\right\}$ is uniformly bounded and equicontinuous on compact subsets of $[a, b)$. Using the Ascoli Theorem we get a subsequence $\left\{v_{n}\right\}$ which converges uniformly on compact subsets of $[a, b)$ to a limit function $u$.

It remains to show that $\left\{v_{n}\right\}$ converges in $L^{2}(m)$. From (6) above

$$
\begin{aligned}
m(x)\left|v_{n}(x)\right|^{2} & \leqq\left(T v_{n}, v_{n}\right) m(x)\left[h_{k}(x, k)\right]^{2} \\
& \leqq M K^{2} m(x)\left[h_{k}(x, k)\right]^{2}, \quad n=1, \cdots .
\end{aligned}
$$

The right side is integrable by hypothesis. The Lebesgue Dominated Convergence Theorem yields $u \in L^{2}(m)$ and $\left\|v_{n}\right\| \rightarrow\|u\|$. Hence,

$$
\left\|v_{n}-u\right\| \rightarrow 0 \text {. }
$$

Hence, $T^{-1}$ is compact, and $T$ has a discrete spectrum. It follows [5, Theorem 2, p. 92] that every selfadjoint extension of $T_{0}$ has a discrete spectrum.

Note that the proof above excludes the case $k=1$. For this case the conclusion of Lemma 1 becomes

$$
\left|u\left(x_{2}\right)-u\left(x_{1}\right)\right| \leqq\left(T_{0} u, u\right)^{1 / 2}\left(\int_{x_{1}}^{x_{2}} \frac{1}{p_{1}(t)} d t\right)^{1 / 2},
$$

and the proof is given in the first step by setting $k=1$. The corresponding result of Lemma 2 (a) becomes

$$
\left|u\left(x_{2}\right)-u\left(x_{1}\right)\right| \leqq(T u, u)^{1 / 2}\left(\int_{x_{1}}^{x_{2}} \frac{1}{p_{1}(t)} d t\right)^{1 / 2}
$$

and the proofs of Lemma 2(a) and the Theorem follow the same pattern for $k=1$ as given above for $k>1$.

4. Remarks. The Theorem provides a test for the spectrum of $T$ to be discrete based on an analysis of $p_{k}(x)$, for $k$ some integer in $[1, n]$. For a test based on $p_{0}(x)$ see [5, Theorem 2, p. 210].

Eastham's technique [3] is based on a comparison to the Euler operator and requires $b=\infty$. However, the procedure used in this paper does not depend on comparison with known results, and the result includes the case of a singularity at a finite endpoint. If $p_{1}(x)=(1+x)^{2+\alpha}, m(x)=1$, $0 \leqq x<\infty$, then both results conclude a discrete spectrum if $\alpha>0$. Eastham obtains a discrete spectrum for

$$
p_{k}(x)=(x+2)^{2 k} \log (x+2), \quad 0 \leqq x<\infty,
$$


but no such conclusion can be drawn from our theorem. It is possible to construct examples of oscillatory $p_{k}(x)$ for which $\lim \inf p_{k}(x) / x^{2 k}$ is finite and $\lim \sup p_{k}(x) / x^{2 k}$ is infinite and, hence, Eastham's results are inconclusive, but for which our theorem concludes a discrete spectrum.

Our theorem, supplemented by Theorem 2, [5, p. 210], yields the results that Friedrichs obtained [4] for those examples for which a discrete spectrum exists. Note that, for the Laguerre operator, a change of variable is needed to obtain the familiar form

$$
t u=-\left(x u^{\prime}\right)^{\prime}+\left(x / 4-\frac{1}{2}\right) u, \quad 0 \leqq x<\infty,
$$

in which case $p_{0}(x)=\left(x / 4-\frac{1}{2}\right) \rightarrow \infty$ as $x \rightarrow \infty$ and the discrete spectrum follows.

\section{REFERENCES}

1. J. V. Baxley, The Friedrichs extension of certain singular differential operators, Duke Math. J. 35 (1968), 455-462. MR 37 \#2036.

2. N. Dunford and J. T. Schwartz, Linear operators. II: Spectral theory. Selfadjoint operators in Hilbert space, Interscience, New York, 1963. MR 32 \#6181.

3. M. S. P. Eastham, The least limit point of the spectrum associated with singular differential operators, Proc. Cambridge Philos. Soc. 67 (1970), 277-281. MR 40 \#5957.

4. K. O. Friedrichs, Criteria for the discrete character of the spectra of ordinary differential operators, Studies and Essays Presented to R. Courant on his Sixtieth Birthday, January 8, 1949, Interscience, New York, 1948, pp. 145-160. MR 9, 353.

5. M. A. Naĭmark, Linear differential operators, GITTL, Moscow, 1954; English transl., Part II, Ungar, New York, 1967. MR 16, 702; MR 41 \#7485.

6. F. Riesz and B. Sz.-Nagy, Leçons d'analyse fonctionnelle, Akad. Kiadó, Budapest, 1953; English transl., Ungar, New York, 1955. MR 15, 132; MR 17, 175.

Department of Mathematics, Georgia Institute of Technology, Atlanta, GEORGIA 30332 